\title{
Bundle structure formation on a polymer film at various temperatures and scanning velocities
}

\author{
X P Wang ${ }^{1,2}$, M M T Loy ${ }^{1}$ and Xudong Xiao ${ }^{1,3}$ \\ ${ }^{1}$ Department of Physics, The Hong Kong University of Science and Technology, \\ Hong Kong, China \\ ${ }^{2}$ Department of Physics, University of Science and Technology of China, \\ Hefei 230026, China \\ E-mail: phxudong@ust.hk
}

Received 11 January 2002, in final form 28 May 2002

Published 21 June 2002

Online at stacks.iop.org/Nano/13/478

\begin{abstract}
The structures formed by atomic force microscopic tip scanning on a $133 \mathrm{~nm}$ thick poly(tert-butyl acrylate) $(\mathrm{P} t \mathrm{BuA})$ film were examined at temperatures ranging from room temperature to $58^{\circ} \mathrm{C}$ with various scanning velocities from 1 to $20 \mu \mathrm{m} \mathrm{s}^{-1}$. It was observed that bumps were created at low temperatures and high scanning velocities. As the temperature was increased and the scanning velocity was decreased, bundles were produced through aggregation of the bumps. The width of the bundles was about $100 \mathrm{~nm}$. As the temperature was further increased to higher than the glassy-rubbery phase transition temperature, $T_{g} \sim 50^{\circ} \mathrm{C}$, of the $\mathrm{P} t \mathrm{BuA}$ polymer, no bundle structures were formed at low scanning velocities. Our results clearly indicate that the formation of bumps and bundles occurs from the interplay of polymer peeling and relaxation. With polymer peeling taking place on soft polymer surfaces, slower relaxation favours the accumulation of polymer coil displacement and the formation of the bump and bundle structures, while fast relaxation results in the recovery of the polymer perturbation and no formation of surface structures.
\end{abstract}

\section{Introduction}

Atomic force microscopy (AFM) in the contact mode is a powerful tool used to manipulate polymer film surfaces on the submicrometre scale in addition to investigation of the topographic features of such films. Recently, many researchers reported that bundle patterns appeared on amorphous polymer surfaces after AFM tip scanning. Most of these bundles were orientated perpendicular to the scanning direction [1-5]. Leung and Goh first found the creation of a periodic pattern with nanometre dimensions as a result of the interaction between the AFM tip and amorphous polystyrene (PS, glassyto-rubbery transition temperature $T_{g} \sim 100{ }^{\circ} \mathrm{C}$ ) [1]. Meyers et al examined PS films at various molecular weights and found bundle-like structures on the film surface. They concluded that the PS surface is not in the glassy state but in the rubbery

3 Author to whom any correspondence should be addressed. state at room temperature [2]. Some further studies have focused on the formation conditions for such structures. For example, Jing et al [3] found that patterns formed more easily in amorphous polyester film $\left(T_{g} \sim 70^{\circ} \mathrm{C}\right)$ than in crystalline film and that the average spatial period of the bundles was proportional to the applied load. Elkaakour et al [4] studied the bundle formation of polyacetylene $\left(T_{g} \sim 200^{\circ} \mathrm{C}\right)$ at various scanning velocities and applied loads, and found that the period of the bundles first decreased and then saturated with the increasing scanning velocity. Peng and Barnes [5] investigated the tacticity effects on the topography of Langmuir-Blodgett films of PMMA $\left(T_{g} \sim 110^{\circ} \mathrm{C}\right)$ and found ellipsoid-like structures (bumps) on the surface without periodicity after initial scanning. In polycarbonate, bump structures were also observed. Moreover, it was confirmed experimentally that there are micropores and cracks in the bumps and that the corresponding elasticity of a film surface with bumps is weakened [6-8]. 
While the formation of ordered or disordered structures on the surface of amorphous polymer films after AFM scanning in the contact mode has been well demonstrated, most of the related research has focused rather more on the structures themselves than on the details of the formation conditions. Most importantly, all these studies were carried out at temperatures much lower than the $T_{g}$ of the bulk polymer. One wonders whether the surface of the amorphous polymer must be in the rubbery state to allow the bump and bundle structures to form [2]. To the best of our knowledge, no investigation on the morphology of polymer film surface had been performed at temperatures close to or higher than the film's bulk $T_{g}$.

In this paper, we investigate whether the soft state is a necessary condition for creating the bundle structures (or other pattern) on an amorphous polymer surface by using variable-temperature AFM. In particular, we study whether the formation of such patterns is related to the glassyrubbery phase transition. With these aims, we performed our experiment on a poly(tert-butyl acrylate) ( $\mathrm{P} t \mathrm{BuA})$ film (bulk $T_{g} \sim 50{ }^{\circ} \mathrm{C}$ ) at temperatures ranging from room temperature $\left(\mathrm{RT} \sim 20^{\circ} \mathrm{C}\right.$ ) to $58^{\circ} \mathrm{C}$. The results showed that bump-like (or bundle-like) structures are created at low temperatures as long as the interaction between the tip and the polymer molecules is sufficient, i.e., the scanning time is rather long. However, due to the fast relaxation of polymer films at temperatures higher than $T_{g}$, the bundle pattern could not be formed at a slow scanning velocity.

\section{Experiment}

$\mathrm{P} t \mathrm{BuA}$ was purchased from Scientific Polymer Products, Inc., Ontario, NY, USA. Its molecular weight distribution was determined as $M_{w}=148 \mathrm{kDa}$ (Da stands for daltons) and $M_{w} / M_{n}=17$ by a Waters gel permeation chromatograph (GPC) system. The bulk $T_{g}$ of the $\mathrm{P} t \mathrm{BuA}$ polymer, measured by a Setaram DSC 92 system at a heating rate of $10^{\circ} \mathrm{C} \mathrm{min}-1$ in dry nitrogen, was about $50^{\circ} \mathrm{C}$. The thin films of $\mathrm{P} t \mathrm{BuA}$ were prepared by spin coating $2 \mathrm{wt} \%$ solution of the polymer in toluene onto a clean silicon substrate at $6000 \mathrm{rpm}$. Prior to the study, the films were annealed in a vacuum oven at $120^{\circ} \mathrm{C}$ overnight. The thickness of the films was about $133 \mathrm{~nm}$ as determined by ellipsometry.

The AFM used in this experiment was home-built, and controlled by RHK electronics (RHK Technology, Troy, MI, USA). The temperature was controlled to $\pm 1^{\circ} \mathrm{C}$ through the application of a DC current to a Peltier heater. A Vshaped silicon nitride integrated cantilever/tip (Parks Scientific Instruments, Sunnyvale, CA, USA) with $0.5 \mathrm{~N} \mathrm{~m}^{-1}$ nominal force constant and $R \sim 50 \mathrm{~nm}$ tip radius was used in the experiment. The measurements were carried out in the conventional contact mode with $10 \mathrm{nN}$ external load force in a low-humidity $(\sim 6 \%)$ environment in order to minimize the capillary effect. The external load was obtained from a forcedistance curve using the $0.5 \mathrm{~N} \mathrm{~m}^{-1}$ nominal force constant supplied by the manufacturer and the sensitivity of the positionsensitive photodiode was calibrated on a hard sample surface.
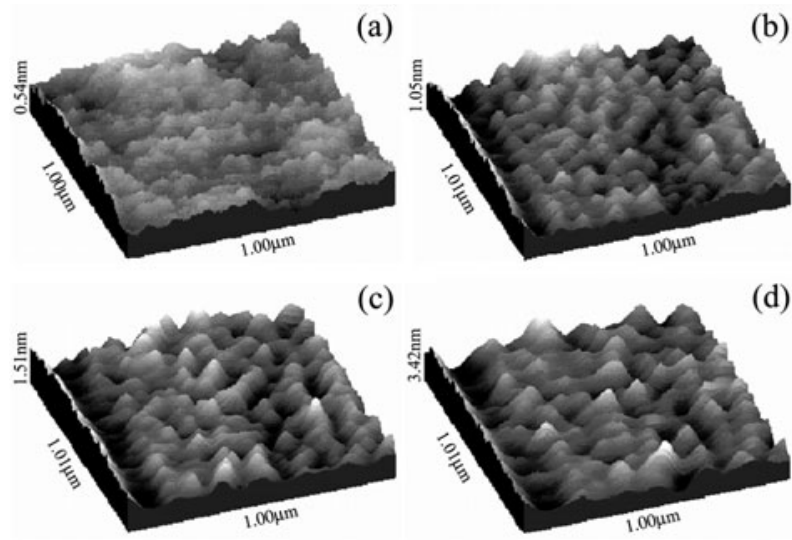

(c)

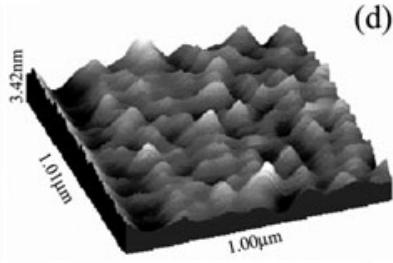

Figure 1. The morphology of the film surface after various numbers of AFM scans at $30^{\circ} \mathrm{C}$ with a $20 \mu \mathrm{m} \mathrm{s}^{-1}$ scanning velocity and a 10 nN load: (a) once, (b) three times, (c) five times and (d) ten times.

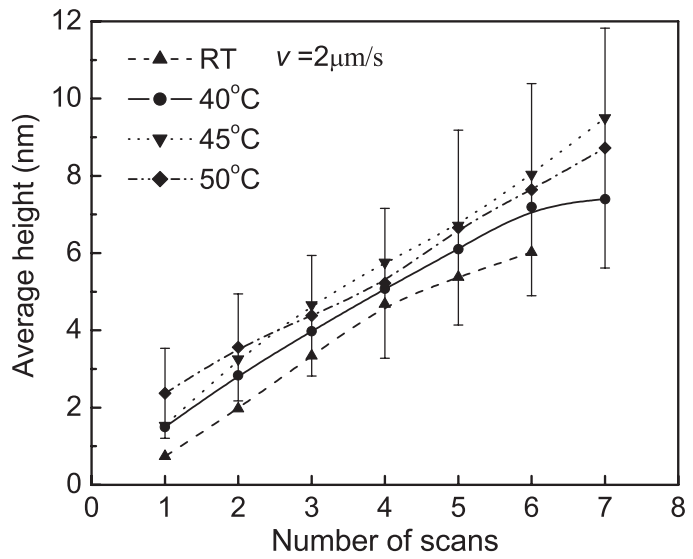

Figure 2. The average height of the surface structures versus the number of AFM scans at various temperatures with a scanning velocity of $2 \mu \mathrm{m} \mathrm{s}^{-1}$.

\section{Results}

In figure 1, the accumulation effect of AFM scanning in creating bumps on the $\mathrm{P} t \mathrm{BuA}$ polymer film surface is shown. The measurements were carried out at a temperature of $30^{\circ} \mathrm{C}$ and a scanning velocity of $20 \mu \mathrm{m} \mathrm{s}^{-1}$. (In this paper, 'bump' refers to the 'zero'-dimensional pile-up structures randomly distributed on the film's surface, while 'bundle' refers to the nearly one-dimensional pile-up structures.) After one scan, the surface remains rather smooth, with an rms roughness of only about $0.2 \mathrm{~nm}$ (figure 1(a)). As scanning is repeated in the same area, randomly distributed non-uniform bumps start to show up and their average size increases gradually. When the number of scans increases from five to ten, the average lateral size of the bumps becomes saturated and the images appear more or less the same, except that the rms roughness value continues to increase from 0.6 to $1.2 \mathrm{~nm}$ (figures 1 (c) and (d)). A similar accumulation effect occurs at other temperatures. In figure 2, we plot the average height of the bumps or bundles formed as a function of the number of scans over the same area for a number of temperatures at a scan velocity of $2 \mu \mathrm{m} \mathrm{s}^{-1}$. Here, the average height is obtained as an averaged value of peak-to-valley distances sampled from 10 section lines/image. Clearly, as the number of scans is increased over the same 

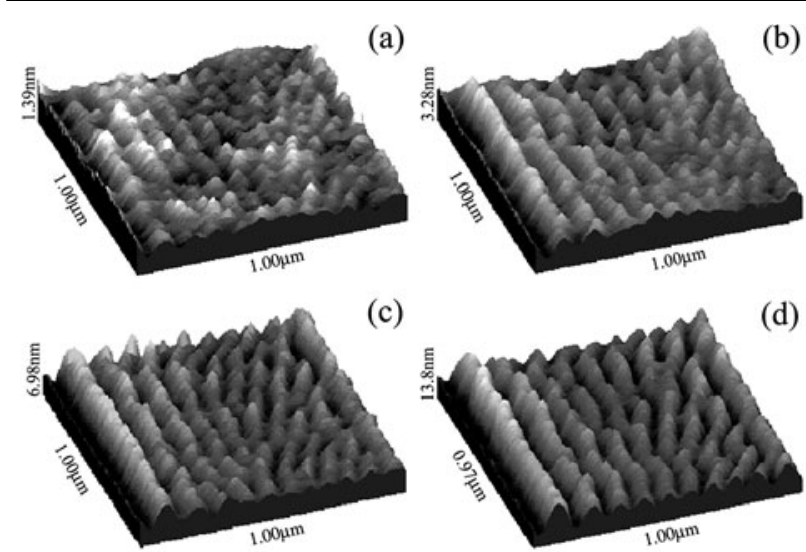

(c)

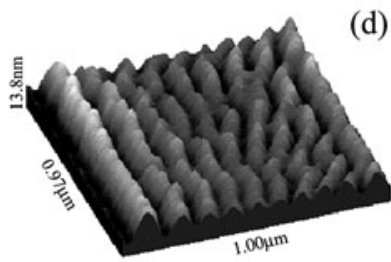

Figure 3. The morphology of the film surface after one scan with a scan velocity of (a) $10 \mu \mathrm{m} \mathrm{s}^{-1}$, (b) $5 \mu \mathrm{m} \mathrm{s}^{-1}$, (c) $2 \mu \mathrm{m} \mathrm{s}^{-1}$ and (d) $1 \mu \mathrm{m} \mathrm{s}^{-1}$ at $40^{\circ} \mathrm{C}$ with a $10 \mathrm{nN}$ load.

area, the height of the structures created increases, indicating a slow relaxation such that the accumulation and the aggregation effects of the perturbation on the polymer molecules of tip rescanning can be observed.

The scanning velocity can also affect the formation of bump and bundle structures. Figure 3 shows the topography of a $\mathrm{P} t \mathrm{BuA}$ polymer surface with various scanning velocities from 1 to $10 \mu \mathrm{m} \mathrm{s}^{-1}$ at $40^{\circ} \mathrm{C}$ with a constant load of $10 \mathrm{nN}$. The periodic bundle structure is ambiguous at $10 \mu \mathrm{m} \mathrm{s}^{-1}$, but starts to form faintly at $5 \mu \mathrm{m} \mathrm{s}^{-1}$ and becomes very clear at a lower scan velocity such as $1 \mu \mathrm{m} \mathrm{s}^{-1}$. On both sides of the images (figures 3(c), (d)), large bundles are also formed, which is possibly the result of the accumulation of materials due to the back-and-forth scanning of the AFM tip. The average period of the bundle structure seems to increase weakly with the decrease in scanning velocity, e.g., from $\sim 100 \mathrm{~nm}$ at $5 \mu \mathrm{m} \mathrm{s}^{-1}$ to $\sim 110 \mathrm{~nm}$ at $1 \mu \mathrm{m} \mathrm{s}^{-1}$. This is consistent with the results reported on polyacetylene, where the bundle width decreases monotonically as the scan velocity goes up to $10 \mu \mathrm{m} \mathrm{s}^{-1}$ and then reaches a plateau [4]. Also in figure 3, the height of the bundle is found to increase from $\sim 0.3$ to $\sim 3 \mathrm{~nm}$ as the scanning velocity decreases from 10 to $1 \mu \mathrm{m} \mathrm{s}^{-1}$. The scanning velocity effect is also observed at other temperatures. In figure 4, the average height of the bundles (or bumps) as a function of scanning velocity for a number of temperatures is plotted. It is obvious that the average height decreases significantly with the scanning velocity. At slow velocities, the structures are clearly formed with large morphological contrast. In general, the higher the temperature, the greater the structure height, with the exception of the case for large numbers of scans, for which the structure height at $45^{\circ} \mathrm{C}$ can exceed that at $50^{\circ} \mathrm{C}$. At fast scanning velocities, e.g., at $20 \mu \mathrm{m} \mathrm{s}^{-1}$, the average structure height is very small and nearly independent of the temperature, indicating that the bump or bundle structures are barely formed.

While the scanning velocity effects on the formation of bump and bundle structures at temperatures lower than $50^{\circ} \mathrm{C}$ are found to be similar, the results at a temperature, $58^{\circ} \mathrm{C}$, higher than the bulk $T_{g}$ are drastically different. As shown in figure 5, weak periodic bundle structures are formed at high scanning velocities $\left(10 \mu \mathrm{m} \mathrm{s}^{-1}\right.$, figure 5(a)). However, in strong contrast to the results at lower temperatures for which

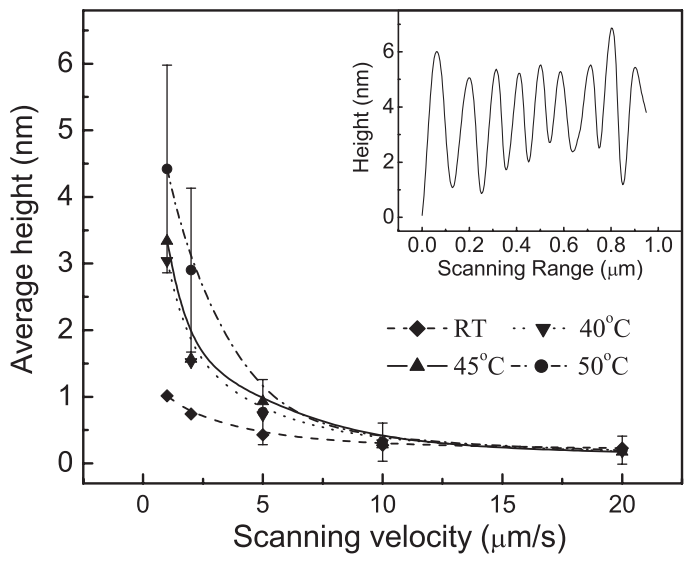

Figure 4. The average height of the surface structures versus the scanning velocity at various temperatures (main panel). The inset shows a typical line profile of the bundle structures.
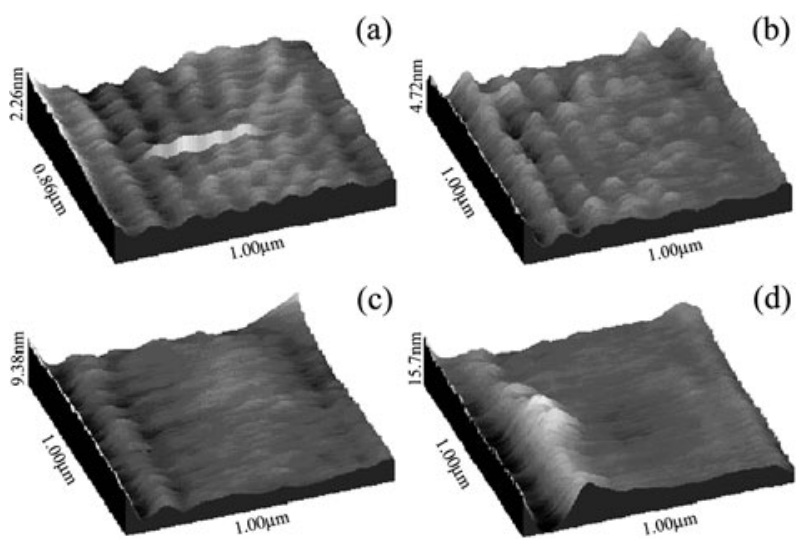

Figure 5. The morphology of the film surface after one scan with a velocity of (a) $10 \mu \mathrm{m} \mathrm{s}^{-1}$, (b) $5 \mu \mathrm{m} \mathrm{s}^{-1}$, (c) $2 \mu \mathrm{m} \mathrm{s}^{-1}$ and (d) $1 \mu \mathrm{m} \mathrm{s}^{-1}$ at $58^{\circ} \mathrm{C}$ with a $10 \mathrm{nN}$ load.

the structures are enhanced at low scanning velocities, the bundle structure at $58^{\circ} \mathrm{C}$ disappears almost completely at low scanning velocities (figures 5(c), (d)).

To further demonstrate the temperature effect on the formation of bundle structures, the morphology of the $\mathrm{P} t \mathrm{BuA}$ polymer surface after one scan at a scanning velocity of $1 \mu \mathrm{m} \mathrm{s}^{-1}$ for various temperatures is shown in figure 6 . When the temperature is very low and the polymer film is most probably in the glassy state, a mixture of the bundle and bump structures is observed (figure 6(a)). As the temperature is increased, the periodic bundle structures start to form clearly, with an average period increasing from $\sim 100 \mathrm{~nm}$ at $40^{\circ} \mathrm{C}$ (figure 6(b)) to $\sim 125 \mathrm{~nm}$ at $50^{\circ} \mathrm{C}$ (figure 6(c)). As the temperature is further increased to above $T_{g}$, no periodic bundle structure can be formed (figure 6(d)). The entire polymer film surface becomes very smooth except for a large bundle on the left side (figures 5(d) and 6(d)), which may be attributed to the transition from static to sliding friction near the edge of the scan area, although the origin of this large bundle is not clearly understood. Moreover, it is found that the molecules in the polymer film at 55 and $58^{\circ} \mathrm{C}$ can be easily peeled and the film surface is worn out after several repetitive scans.

It is interesting to compare the results at 50 and $58^{\circ} \mathrm{C}$. The period of the bundle structures formed at $58^{\circ} \mathrm{C}$ at $10 \mu \mathrm{m} \mathrm{s}^{-1}$ 
(a)
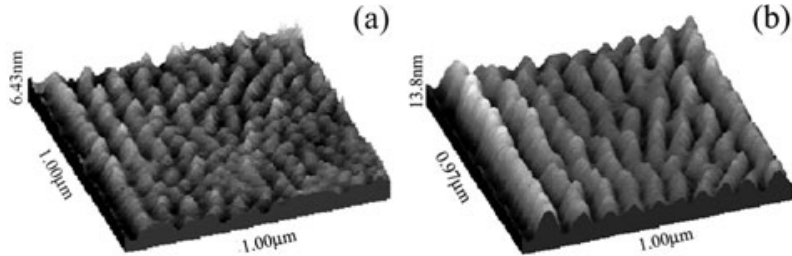

(c)
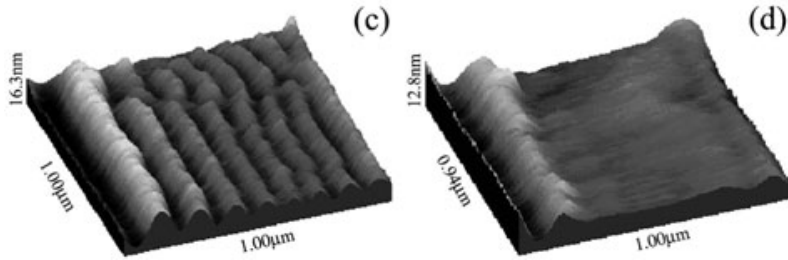

Figure 6. The morphology of the film surface after one scan at (a) $20^{\circ} \mathrm{C}$, (b) $40^{\circ} \mathrm{C}$, (c) $50^{\circ} \mathrm{C}$ and (d) $55^{\circ} \mathrm{C}$ with a scanning velocity of $1 \mu \mathrm{m} \mathrm{s}^{-1}$ and a $10 \mathrm{nN}$ load.

(figure 5(a)) is about the same as that of those formed at $50^{\circ} \mathrm{C}$ at $1 \mu \mathrm{m} \mathrm{s}^{-1}$ (figure 6(c)) except that the height of the bundles is much larger for the latter case. This phenomenon might be explained by the principle of equivalence between the scanning velocity and the temperature for the viscoelastic properties of the polymer, which states that the effect of a scan velocity increase is equivalent to a temperature decrease $[9,10]$. The equivalence principle results from the relaxation of polymers. It is well known that, on the one hand, the higher the temperature, the faster the molecular relaxation, while, on the other hand, the degree of relaxation of the molecules depends on the relaxation time, i.e., the longer the waiting time, the more complete the relaxation. Thus, a relaxation process performed at a high temperature for a short time (or a fast scan velocity in the present experiment) is equivalent to one at low temperature for a longer time (or slow scan velocity here). The equivalence between the scanning velocity and the temperature can be further confirmed in figure 7. By comparing the morphologies between figures 7(a) and (b), we find that the result after one scan at $40^{\circ} \mathrm{C}$ at $1 \mu \mathrm{m} \mathrm{s}^{-1}$ is similar to that after one scan at $45^{\circ} \mathrm{C}$ at $5 \mu \mathrm{m} \mathrm{s}^{-1}$. Even after five scans, the equivalence can still be demonstrated (figures 7(c) and (d)).

\section{Discussion}

The formation of bumps and bundles is an interplay of polymer peeling and polymer relaxation. In the peeling process, a strip of polymer (possibly formed by entangled polymer coils) is laterally pushed ahead of the AFM cantilever tip as the AFM tip scans over a polymer surface. Because of the relatively large size of the polymer molecules, the molecular displacement extends to a larger area than the tip/sample contact; that is, the perturbation of the polymer molecular positions due to the present line scan can extend to the next few scan lines in a twodimensional image scan. With sufficiently slow relaxation, the displaced polymer molecules would remain in their displaced positions when the AFM scans the next line. This displacement effect can then be accumulated to form bumps and, in some cases, even correlated to form bundles.

Assuming that the molecules of $\mathrm{P} t \mathrm{BuA}$ are in their equilibrium conformations and the molecular chains are mostly
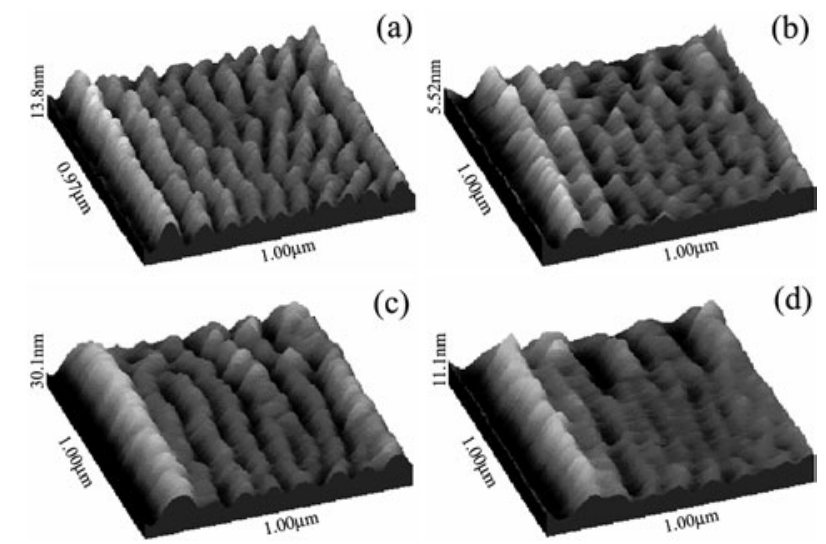

Figure 7. The morphology of the film surface after one scan at (a) $40{ }^{\circ} \mathrm{C}$ with $1 \mu \mathrm{m} \mathrm{s}^{-1}$, (b) $45^{\circ} \mathrm{C}$ with $5 \mu \mathrm{m} \mathrm{s}^{-1}$, and after five scans at (c) $40{ }^{\circ} \mathrm{C}$ with $1 \mu \mathrm{m} \mathrm{s}^{-1}$, (d) $45^{\circ} \mathrm{C}$ with $5 \mu \mathrm{m} \mathrm{s}^{-1}$.

in coil shapes [11], the root mean square radius of such a coil can be estimated from [3]

$$
\begin{gathered}
r_{w}=\sqrt{0.096 \times 10^{-20}\left(M_{w} \times 10^{3}\right)^{0.98}} \\
\sim 10.6 \mathrm{~nm} \quad\left(M_{w} \sim 148 \mathrm{kDa}\right) .
\end{gathered}
$$

Since $r_{w}$ is much smaller than the polymer film thickness $(133 \mathrm{~nm})$, the interface confinement effect may be neglected and the as-spun polymer film can be simply considered as consisting of random coils. With the average diameter of bumps being $\sim 100 \mathrm{~nm}$ and the bump height being close to the coil dimension (see figure 1), each bump is estimated to consist of $\sim 100$ polymer molecular coils. For scans faster than $10 \mu \mathrm{m} \mathrm{s}^{-1}$, the molecular coils do not displace significantly due to the polymer's slower response and thus no large bumps are formed (see figure 4). The results from figures 1-4 also indicate that the polymer surface has to be soft enough that the peeling of the polymer coils can take place from the AFM tip scanning in order to form the bump or bundle structures. The dependence on temperature - the higher the temperature, the greater the average height at a given scan velocity or number of scans-clearly supports this claim.

However, the softness of the polymer surface is not a sufficient condition for the formation of bump and bundle structures. Our experimental results indicate that the bundles or bumps cannot be formed at a scanning velocity of $1 \mu \mathrm{m} \mathrm{s}^{-1}$ when the temperature is above $55^{\circ} \mathrm{C}$, clearly in the soft state. One possible reason for this is that the peeled polymers pile up outside the scanning zone, leading to no bundle or bump formation. Scanning over a larger area at a much lower load $(0.5 \mathrm{nN}$, figure 8$)$ shows no extra materials accumulation beyond the original scan area, indicating the unlikeliness of this interpretation. There are two other possible explanations for this observation. The first is that the polymer relaxation is so fast that most of the polymer coils displaced by the scanning would return towards their equilibrium positions in order to relax the accumulated mechanical stress (such as in the stretched polymer strands of the displaced polymer coils) upon the removal of the AFM tip. The second possibility is that the period of the bundles is so wide that it exceeds the scope of the image. In order to identify which of the two reasons is more likely, we perform a calculation of the bundle period as a function of temperature following the model proposed in [4]. 


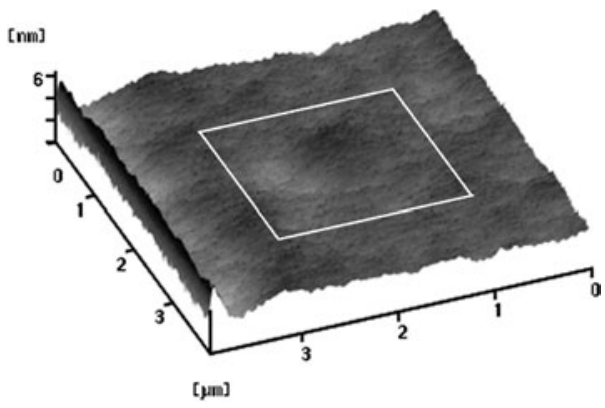

Figure 8. The zoom-out image $(4 \mu \mathrm{m} \times 4 \mu \mathrm{m})$ of the $\mathrm{P} t \mathrm{BuA}$ film at $0.5 \mathrm{nN}$ after scanning at the centre $(2 \mu \mathrm{m} \times 2 \mu \mathrm{m})$ at $10 \mathrm{nN}$. The scanning is carried out at $60^{\circ} \mathrm{C}$, and the thermal drift is $<0.1 \mu \mathrm{m}$.

In this model, the polymer surface is assumed to be first peeled and pushed forward by the tip and then aggregated together ahead of the tip to form bundles. As a result, a strain, $\Delta / l$, can be yielded in the polymer and the elastic energy stored in the film can induce a tangential force, $F_{t}$, that repels the tip apex. $F_{t}$ can be expressed by $[4,12]$

$$
F_{t} \propto E a h \Delta / l,
$$

where $E, a, l$ and $h$ are the Young's modulus, the contact radius between the tip and the polymer, the peeled polymer length and the tip penetration depth into the polymer, respectively. Following equation (1), $F_{t}$ increases with the strain, $\Delta / l$, as the tip continues scanning. As soon as $F_{t}$ reaches a critical value, $F_{t}^{s}$, which is equal to the product of the frictional coefficient, $\mu$, and the total load force, $L_{t}$ (the sum of the external load $L$ and the adhesive force $F_{a}$ ) [4], the tip no longer peels the polymer molecules and thus slides over the piled polymer coils that form the bundle or bump. This phenomenon is to some extent similar to the stick-slip feature in atomic friction measurements.

Taking into account the adhesion force, $F_{a}=3 \pi \gamma R / 2$, the radius of the contact area $a$ is given by the JKR formula $[13,14]$

$$
\begin{aligned}
a^{3} \frac{3 K}{4 R} & =L+3 \pi \gamma R+\sqrt{6 \pi \gamma R L+(3 \pi \gamma R)^{2}} \\
& =\left(\sqrt{L+F_{a}}+\sqrt{F_{a}}\right)^{2},
\end{aligned}
$$

where $K$ is the reduced elastic modulus $\left(K^{-1}=\left(1-v_{1}^{2}\right) / E_{1}+\right.$ $\left.\left(1-v_{2}^{2}\right) / E_{2}\right)$ and $R$ is the radius of the tip. The penetration depth, $h$, is given as $h=R-\sqrt{R^{2}-a^{2}}$ for a spherical tip and can be calculated once $a$ is known. With the adhesion force, $F_{a}$, and the elastic modulus, $E_{2}$, of the polymer film measured as a function of temperature [15] (see the inset of figure 9), following Elkaakour et al [4], the bundle period can be calculated. The results are plotted in the main panel of figure 9. (In the calculation, the friction coefficient is presumed to be independent of the temperature, the tip elastic modulus, $E_{1}$, is $\sim 155 \mathrm{GPa}$ and the Poisson ratios, $v_{1}, v_{2}$, are 0.5.) The observed bundle periods from our experiment are also plotted in the figure as squares. The agreement is reasonable in the relevant temperature range. From the calculation, we notice that the bundle period has a strong dependence on the adhesion, which dictates the behaviour in the bundle period as the temperature increases. Below $45^{\circ} \mathrm{C}$, the adhesion is nearly constant as is the bundle period. Above $45^{\circ} \mathrm{C}$, the adhesion force increases as does the bundle period. As the

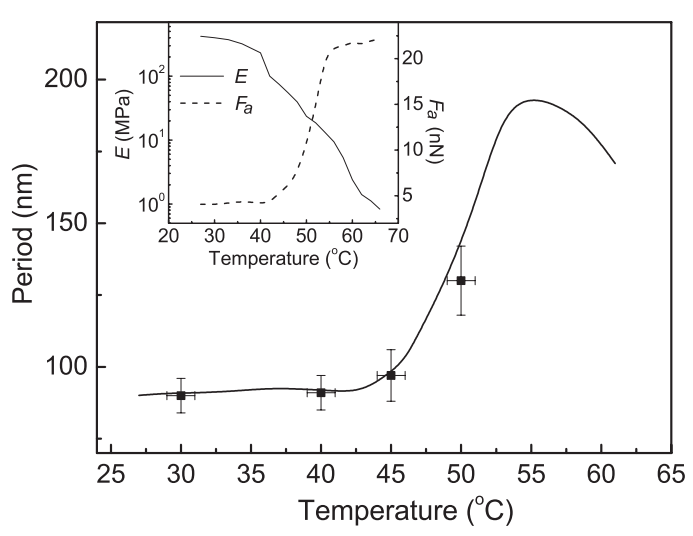

Figure 9. Main panel: temperature dependence of the calculated bundle period (line) and the experimental data (squares). Inset: temperature dependence of the adhesion force (dashed line) and Young's modulus (curve). Scanning velocity: $1 \mu \mathrm{m} \mathrm{s}^{-1}$; external load: $10 \mathrm{nN}$.

temperature further increases above $55^{\circ} \mathrm{C}$, the decrease of the polymer elastic modulus finally dominates and contributes to the decrease in the bundle period. Although the validity of the model is questionable, particularly in the rubbery state, we believe the calculated temperature dependence of the bundle period to be a good guideline.

From figure 9, we see that the bundle period is predicted to be $\sim 200 \mathrm{~nm}$ from 55 to $58^{\circ} \mathrm{C}$, well within the scope of the AFM images. Thus, the disappearance of the bundles at high temperatures is not caused by the large bundle period. Rather, the cause must be the fast relaxation of the polymers at these temperatures. The previous adhesion measurements demonstrate that the $\mathrm{P} t \mathrm{BuA}$ film at these temperatures has been transformed to the rubbery state [15], which definitely shortens the polymer relaxation time. As the polymer coils are perturbed from their original positions when the AFM tip scans at the $J$ line, the resulting perturbation of the polymer coils at the $J+1$ line will relax back towards the original position before the AFM tip scans at the $J+1$ line. Therefore, no accumulation effect would occur and no bundles or even bumps can be formed in the rubbery states when the scan velocity is slow. From the temperature dependence measurement, we conclude that the formation of bundles depends on the competition between the softness of the polymer, which results in polymer peeling, and the speed of the relaxation, which dictates the accumulation effect of the displacement of the polymer coils.

\section{Summary}

We have studied the formation of bumps and bundles on a $133 \mathrm{~nm}$ thick $\mathrm{P} t \mathrm{BuA}$ polymer film at various temperatures and scanning velocities using AFM in the contact mode. The results demonstrated that the bundle or bump structures can be produced predominantly in the glassy state when the material has a slow relaxation but is soft enough for polymer coil displacement. Due to the equivalence of the scanning velocity and the temperature of the polymer viscoelasticity, both the scanning velocity and the temperature can influence the formation of the bundle structures, i.e., the smaller the velocity (or the higher the temperature), the wider the bundle period. On the other hand, the bundles hardly form in the 
rubbery state when the relaxation time is short and the polymer coils displaced by the AFM tip can quickly recover to their equilibrium positions.

\section{Acknowledgments}

We wish to acknowledge the financial support of the Hong Kong University of Science and Technology through the William Mong Solid State Cluster Laboratory, the HKUST Research Infrastructure Grant and the High Impact Area Fund as well as from the Research Grants Council of Hong Kong through Grant No HKUST6139/97P.

\section{References}

[1] Leung M and Goh C 1992 Science 25564

[2] Meyers G F, DeKoven B M and Seitz J T 1992 Langmuir 82330
[3] Jing J, Henriksen P N, Wang H and Marteny P 1995 J. Mater. Sci. 305700

[4] Elkaakour Z, Aime J P, Bouhacina T, Odin C and Masuda T 1994 Phys. Rev. Lett. 733231

[5] Peng J B and Barnes G T 1996 Thin Solid Films 284-5 444

[6] Hamada E and Kaneko R 1992 J. Phys. D: Appl. Phys. 25 A53

[7] Khurshudov A and Kato K 1997 Wear 205

[8] Iwata F, Matsumoto T and Sasaki A 2000 Nanotechnology 11 10

[9] Flory P J 1953 Principles of Polymer Chemistry (Ithaca, NY: Cornell University Press)

[10] Gedde U W 1995 Polymer Physics (London: Chapman and Hall)

[11] Kirste R G, Kruse W A and Ibel K 1975 Polymer 16120

[12] Barquins M 1984 J. Appl. Polym. Sci. 293041

[13] Johnson K L, Kendall K and Roberts A D 1971 Proc. R. Soc. A 324301

[14] Bhushan B (ed) 1996 Micro/Nanotribology and its Applications (Dordrecht: Kluwer-Academic)

[15] Tsui O K C, Wang X P, Jacob Y L Ho, Ng T K and Xiao X D 2000 Macromolecules 334198 